\title{
Transarterial chemoembolization as salvage therapy after unsuccessful hepatic arterial infusion chemotherapy in advanced hepatocellular carcinoma
}

\author{
KAZUTA FUKUMORI ${ }^{1}$, YOICHI YANO ${ }^{2}$, EIJI ANDO ${ }^{1}$, SHUJI SUMIE ${ }^{1}$, KOTORO KUWAKI $^{1}$, \\ FUMIHIKO YAMASHITA ${ }^{2}$, MASATOSHI TANAKA ${ }^{1}$ and MICHIO SATA ${ }^{1}$ \\ ${ }^{1}$ Division of Gastroenterology, Department of Internal Medicine, Kurume University School of Medicine, \\ 67 Asahi-machi, Kurume-shi, Fukuoka-ken 830-0011; ${ }^{2}$ Department of Internal Medicine, Saga \\ Social Insurance Hospital, 3-8-1 Hyogo-Minami, Saga-shi, Saga-ken 849-8522, Japan
}

Received January 14, 2008; Accepted March 31, 2008

\begin{abstract}
The prognosis for advanced hepatocellular carcinoma (HCC) remains unsatisfactory. Transarterial chemoembolization (TACE) and/or hepatic arterial infusion chemotherapy (HAIC) have been reported to be useful options. However, there are few reports of salvage therapies for patients without a curative response to initial chemotherapy. The aim of this study was to elucidate the efficacy of additional TACE as salvage therapy in cases of advanced HCC which failed to respond to HAIC. Of 43 patients with advanced HCC who did not show a complete response (CR) to HAIC, 12 were treated with additional TACE as salvage therapy (Group A). The rest were enrolled as disease control subjects (Group B). Response rates and prognosis were compared. For HAIC, cisplatin (10 mg/body on days 1-5) was administered. Subsequent treatment was the infusion of 5-fluorouracil (250 mg/body on days 1-5), which was scheduled for 4 serial courses. For TACE, carboplatin (150 mg/body) or epirubicin $(30 \mathrm{mg} /$ body) was administered mixed with $3 \mathrm{ml}$ of ethiodized oil every 4 weeks. A CR or PR, ST and PD were observed in 6, 3, and 3 patients in Group A and 13, 18 and 0 patients in Group $\mathrm{B}$, respectively. The difference in response between the two groups was significant ( $\mathrm{P}=0.0074)$. The 1-, 2- and 3-year survival rates were 83.3, 75.0 and $44.4 \%$ in Group A and 83.9, 41.5 and $11.3 \%$ in Group B, respectively. Patients in Group A had a better prognosis than did those in Group $\mathrm{B}(\mathrm{P}=0.018)$. Median survival was 31.9 months (5.8-41.1) in Group A and 16.2 months (3.3-53.2) in Group B. Consequently, TACE as
\end{abstract}

Correspondence to: Dr Kazuta Fukumori, Division of Gastroenterology, Department of Internal Medicine, Kurume University School of Medicine, 67 Asahi-machi, Kurume-shi, Fukuoka-ken 830-0011, Japan

E-mail: kazuta.f@d8.dion.ne.jp

Key words: hepatocellular carcinoma, transarterial chemoembolization, hepatic arterial infusion chemotherapy, additional therapy salvage therapy after HAIC may improve the prognosis for patients with advanced HCC.

\section{Introduction}

Hepatocellular carcinoma (HCC) is one of the most common cancers in the world. While surveillance programs for chronic liver diseases have led to early diagnosis and effective treatments, the prognosis for advanced HCC remains unsatisfactory. Transarterial chemoembolization (TACE) has been shown to be a palliative treatment with evidence of survival benefit $(1,2)$. Recently, improvements in an implantable drug delivery system have enabled repeated arterial infusion of chemotherapeutic agents for patients with advanced HCC. Although the optimal doses, schedule and types of chemotherapy used in conjunction with this system have not been fully established, an improvement in response rates and survival has been reported (3-8). We reported on the usefulness of hepatic arterial infusion chemotherapy (HAIC) using cisplatin (CDDP) and 5-fluorouracil (5-FU) for advanced HCC with portal vein tumor thrombosis (PVTT) $(9,10)$. However, there are few reports on the best salvage treatment for patients who do not achieve a complete response to the initial chemotherapy. The aim of this study was to elucidate whether additional TACE as salvage therapy after unsuccessful HAIC affects the prognosis of patients with advanced HCC.

\section{Patients and methods}

Patients. Ninety-two patients with advanced HCC were treated with HAIC using CDDP and 5-FU via a subcutaneouslyimplanted injection port at Saga Social Insurance Hospital between June 1990 and December 1997. HCC was diagnosed clinically using imaging studies including ultrasonography, computed tomography (CT), magnetic resonance imaging and celiac angiography, and tumor biopsy. Due to the advanced tumor stage or the coexistence of cirrhosis, these patients were not suitable for surgical resection or ablation therapy, such as percutaneous ethanol injection, microwave coagulation therapy or radio-frequency tumor ablation. The 11 patients who achieved a complete response (CR) to HAIC, 21 patients 
Table I. Patient characteristics.

\begin{tabular}{|c|c|c|c|}
\hline & Group A & Group B & P-value \\
\hline Number & 12 & 31 & \\
\hline Gender (male/female) & $10 / 2$ & $25 / 6$ & 0.805 \\
\hline Age $(\text { years })^{\mathrm{a}}$ & $65.2 \pm 8.8$ & $64.5 \pm 8.6$ & 0.843 \\
\hline Virus marker (anti-HCV/HBs Ag) & $12 / 0$ & $27 / 4$ & 0.200 \\
\hline Child's classification $^{\mathrm{b}}(\mathrm{A} / \mathrm{B} / \mathrm{C})$ & $8 / 4 / 0$ & $19 / 12 / 0$ & 0.705 \\
\hline Tumor size $(\mathrm{mm})^{\mathrm{a}}$ & $41.8 \pm 21.5$ & $53.6 \pm 22.6$ & 0.126 \\
\hline Tumor stage $(\mathrm{II} / \mathrm{III}+\mathrm{IV})$ & $3 / 9$ & $9 / 22$ & 0.785 \\
\hline Serum $\alpha$-fetoprotein $(\mathrm{ng} / \mathrm{ml})^{\mathrm{a}}$ & $5,128 \pm 13,488$ & $2,463 \pm 7,549$ & 0.414 \\
\hline Serum des-g-carboxy prothrombin $(\mathrm{mAU} / \mathrm{ml})^{\mathrm{a}}$ & $2,222 \pm 6,928$ & $3,282 \pm 11,744$ & 0.771 \\
\hline Pre-treatment (none/PEIT/TACE/MCT/resection) & $7 / 2 / 2 / 1 / 0$ & $10 / 6 / 10 / 4 / 1$ & 0.168 \\
\hline
\end{tabular}

${ }^{a}$ Data are the mean $\pm \mathrm{SD} .{ }^{\mathrm{b}}$ Based on the criteria of Child and Turcotte (11). ${ }^{\mathrm{C} A c c o r d i n g}$ to TMN classification (18). Anti-HCV, antibody for hepatitis C virus; HBs Ag, hepatitis B surface antigen; PEIT, percutaneous ethanol injection therapy; TACE, transarterial chemoembolization; MCT, microwave coagulation therapy; HAIC, hepatic arterial infusion chemotherapy.

who developed PVTT and 17 patients whose hepatic reserve was classified as Child's classification Grade $\mathrm{C}$ were excluded from the study (11). The remaining 43 patients were enrolled and divided into two groups: Group A, 12 patients treated with additional TACE as salvage, and Group B, 31 patients who did not receive TACE. Table I summarizes the clinical profiles of the patients in each group. There were no significant differences in the clinical profiles between the two groups.

Technique of catheter placement. A catheter was inserted through the femoral artery using the Seldinger method. Following the detection of HCC, an indwelling 4- or 5-French heparin-coated catheter was put in place, with the tip of the catheter being inserted in the common or proper hepatic artery. The right gastric artery and the gastroduodenal artery were occluded with steel coils to prevent any gastroduodenal injury following administration of the anticancer agents. The other end of the catheter was connected to an injection port, and the device was implanted into a subcutaneous pocket in the lower right quadrant. The entire procedure was performed under local anesthesia. In order to prevent the occlusion of the device, $5 \mathrm{ml}$ (5000 units) of heparin solution was injected biweekly.

Chemotherapeutic regimen of HAIC. One course of HAIC consisted of the daily administration of CDDP $(10 \mathrm{mg} / \mathrm{h}$ on days 1-5) followed by the infusion of 5-FU (250 $\mathrm{mg} / 5 \mathrm{~h}$ on days 1-5) using a mechanical infusion pump via a subcutaneously-implanted injection port. In principle, the patients were to receive HAIC for four serial courses every 6 weeks. A serotonin antagonist, andansetron hydrochloride, was administered intravenously as an antiemetic, and saline infusion $(500 \mathrm{ml})$ was administered during the chemotherapy infusion.

Transarterial chemoembolization as salvage therapy. TACE was administered as salvage therapy to those patients who consented to additional therapy and whose response to HAIC was classified as partial response (PR), stable disease (ST) or progressive disease (PD). A lipiodol emulsion, $3 \mathrm{ml}$ of ethiodized oil (Lipiodol), carboplatin (150 mg/body) or epirubicin (30 mg/body), was injected slowly through the indwelling injection port under fluoroscopy. TACE was repeated every 4 weeks until one of the following signs was noted: i) disappearance of all viable HCC on enhanced CT, ii) an apparent increase in the tumor diameter or the appearance of portal vein tumor thrombosis, or iii) deterioration of hepatic function. The total doses of carboplatin and epirubicin were set at $1500 \mathrm{mg}$ and $300 \mathrm{mg}$, respectively. In this study, a gelatin sponge was not used in order to avoid the deterioration of liver function $(8,12)$.

Response criteria. Tumor response to HAIC was evaluated in both groups, and tumor response to TACE was evaluated in Group A. Local response to treatment was classified based on the criteria defined by the World Health Organization: $\mathrm{CR}$, complete disappearance of all known disease and the appearance of no new lesions as determined by two observations not less than 4 weeks apart; $\mathrm{PR},>50 \%$ reduction in the total tumor load of all measurable lesions determined by two observations not less than 4 weeks apart; ST, does not qualify as $\mathrm{CR} / \mathrm{PR}$ or PD; and PD, a $>25 \%$ increase in the size of one or more measurable lesions or the appearance of one or more new lesions.

Adverse events. Adverse events were graded according to the Common Terminology Criteria for Adverse Events v3.0 (13).

Statistical analysis. Data were expressed as the means \pm SD. The $\chi^{2}$ test, Fisher's exact probability test, the Student's t-test and Welch's t-test were used to compare patient characteristics and tumor response to HAIC. Cumulative survival rates were calculated using the Kaplan-Meier method. Statistical differences were considered significant at $\mathrm{P}<0.05$.

This study was approved by the Ethics Committee of the Saga Social Insurance Hospital. 
Table II. Response to treatment.

\begin{tabular}{|c|c|c|c|}
\hline & \multicolumn{2}{|c|}{ Group A $(n=12)$} & \multirow{2}{*}{$\frac{\text { Group B }(n=31)}{\text { HAIC }}$} \\
\hline & HAIC & HAIC and TACE & \\
\hline CR & 0 & 1 & 0 \\
\hline PR & 3 & 5 & 13 \\
\hline ST & 8 & 3 & 18 \\
\hline PD & 1 & 3 & 0 \\
\hline \multirow{2}{*}{ P-value } & \multicolumn{3}{|c|}{0.0074} \\
\hline & \multicolumn{2}{|c|}{0.483} & \\
\hline
\end{tabular}

Abbreviations as in Table I. Group A, patients receiving HAIC followed by TACE; Group B, patients receiving HAIC only. CR, complete response; PR, partial response; ST, stable disease; PD, progressive disease.

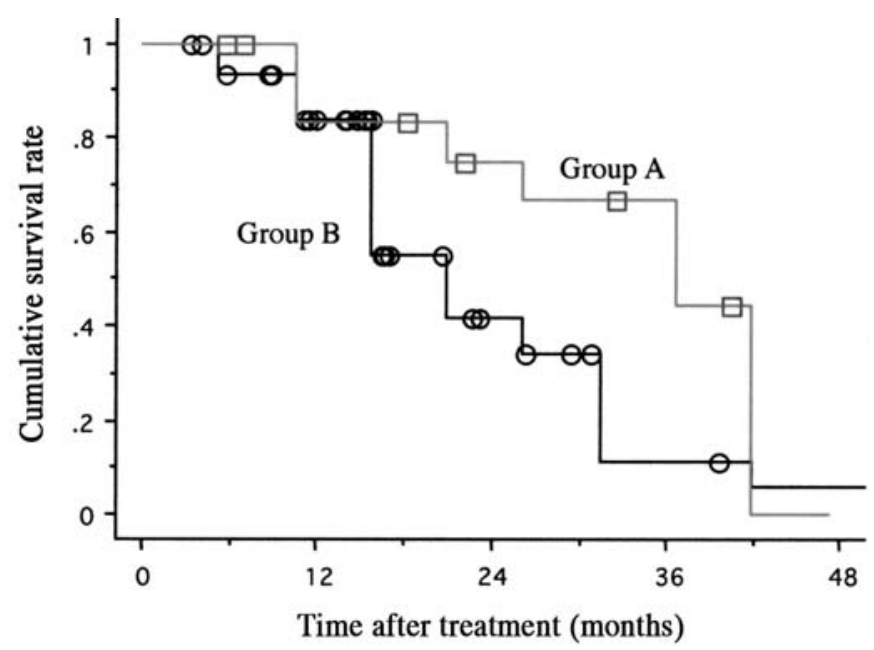

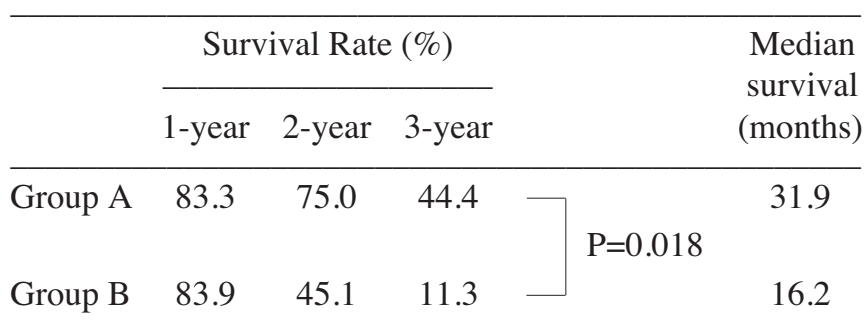

Figure 1. The cumulative survival rate of patients with advanced hepatocellular carcinoma who received hepatic arterial infusion chemotherapy only (Group B, $\mathrm{n}=31$ ) and those who received the same treatment followed by transcatheter arterial chemoembolization (Group $\mathrm{A}, \mathrm{n}=12$ ). The survival rate was significantly longer for patients in Group A than in Group B ( $\mathrm{P}=0.018)$.

\section{Results}

The patients in Groups A and B received 5.75 \pm 2.60 and $5.89 \pm 3.30$ courses of HAIC, respectively. TACE was performed for $5.66 \pm 1.9$ courses in the Group A patients. As shown in Table II, CR+PR, ST and PD were observed in 6, 3 and 3 patients in Group A and 13, 18 and 0 patients in Group B,
Table III. Response to treatment.

\begin{tabular}{|c|c|c|}
\hline & $\begin{array}{c}\text { Group A } \\
(\mathrm{n}=12)\end{array}$ & $\begin{array}{c}\text { Group B } \\
(\mathrm{n}=31)\end{array}$ \\
\hline \multicolumn{3}{|l|}{ Complications } \\
\hline Catheter obstruction & $2(16)$ & $4(13)$ \\
\hline $\begin{array}{l}\text { Hematoma around } \\
\text { injection port }\end{array}$ & $1(8)$ & $5(16)$ \\
\hline $\begin{array}{l}\text { Dislocation of the } \\
\text { tip of the catheter }\end{array}$ & $1(8)$ & $2(6)$ \\
\hline $\begin{array}{l}\text { Infection around } \\
\text { injection port }\end{array}$ & $1(8)$ & $7(22)$ \\
\hline $\begin{array}{l}\text { Obstruction of } \\
\text { hepatic artery }\end{array}$ & 0 & 1 (3) \\
\hline \multicolumn{3}{|l|}{ CTCAE } \\
\hline Nausea & Grade 1, 2 (16) & Grade 1, 6 (19) \\
\hline Anorexia & 0 & Grade 2,1 (3) \\
\hline Mucositis-stomach & Grade 1, 1 (8) & Grade 1,2 (6) \\
\hline Ulcer, GI-stomach & 0 & Grade 2, 4 (13) \\
\hline Ulcer, GI-duodenum & Grade 2, 1 & Grade 2,2 (6) \\
\hline Fever $^{\mathrm{a}}$ & Grade 1, 1 (8) & 0 \\
\hline Diarrhea & 0 & Grade 1,1 (3) \\
\hline
\end{tabular}

Data are expressed as the numbers of patients and (percentages). CTCAE, Common Terminology Criteria for Adverse Events v3.0 (13). ${ }^{\text {aPeculiar to TACE. }}$

respectively. The difference in tumor response between the two groups was significant $(\mathrm{P}=0.0074)$. Three patients in Group A responded to TACE although they had failed to respond to $\mathrm{HAIC}$, including one $\mathrm{CR}$ case.

As shown in Fig. 1, the 1-, 2- and 3-year survival rates were $83.3,75.0$ and $44.4 \%$ in Group $\mathrm{A}$ and $83.9,41.5$ and $11.3 \%$ in Group B, respectively. Patients in Group A had a better prognosis than did those in Group B $(\mathrm{P}=0.018)$. The median survival was 31.9 months (5.8-41.1) in Group A, and 16.2 months (3.3-53.2) in Group B.

No severe adverse reactions or complications, such as hepatic abscess, severe myelosuppression or severe hepatic damage, occurred (Table III), although some patients needed minimal symptomatic treatments.

\section{Discussion}

We evaluated the efficacy of additional TACE as a salvage therapy for patients with advanced HCC who did not achieve a complete response to HAIC. Since we did not use a gelatin sponge to avoid deterioration of hepatic reserve, the TACE employed in this study may be different from conventional TACE.

As for the administration of Lipiodol emulsion mixed with anticancer agents, its antitumor mechanisms have been reported to be the microembolization of tumor vessels and a slow release of anticancer agents (14-16). As shown in Table II, the tumor response rate (CR+PR for all cases) in Group A was comparable to that of Group B; the former was 
$50 \%$ and the latter $42 \%$. However, patients in Group A had a better prognosis than did those in Group B; the median survival was 31.9 months in Group A and 16.2 months in Group B (Fig. 1). The exact reason for this observation is not at present clear. Repetitive microembolization of a tumorfeeding artery, which is unrelated to the drug sensitivities of the tumor cells, may retard the progression of $\mathrm{HCC}$, although complete necrosis of the tumor is rare. Preservation of the hepatic arteries and hepatic reserve, which may have resulted from performing the treatments without a gelatin sponge, may also have played an important role in permitting repeated anticancer treatments. Three patients in Group A, considered to have ST following the administration of HAIC, responded to this salvage therapy and achieved CR or PR. While effective treatment for advanced $\mathrm{HCC}$ has not been established, particularly in patients without curative responses to initial chemotherapy, TACE without a gelatin sponge may prove to be a useful second therapeutic alternative.

However, in two patients in this study additional TACE resulted in a worsening of tumor stage. Akamastu et al (17) reported that TACE could lead to the development of poorlydifferentiated HCC or multiple recurrences. TACE seems to be a double-edged sword when used in cases of advanced HCC. Consequently, after the administration of TACE, follow-up studies are needed for the early detection of HCC progression.

No severe adverse events related to TACE were observed, although the sample size of this study was small. In terms of quality of life issues, it was significant that all the patients who received TACE could be treated on an outpatient basis.

In conclusion, repetitive cycles of TACE without a gelatin sponge may be a useful salvage therapy for patients with advanced HCC who do not have portal vein tumor thrombosis. However, the use of both HAIC and TACE in advanced HCC has not been confirmed for populations worldwide. A randomized prospective control trial is required to confirm these findings.

\section{References}

1. Llovet JM, Real MI, Montana X, et al: Arterial embolization or chemoembolization versus symptomatic treatment in patients with unresectable hepatocellular carcinoma: a randomized control trial. Lancet 359: 1734-1739, 2002.

2. Lo CM, Ngan H, Tso WK, et al: Randomized controlled trial of transarterial lipiodol chemoembolization for unresectable hepatocellular carcinoma. Hepatology 35: 1164-1171, 2002.
3. Patt YZ, Charnsangavei C, Yoffe B, et al: Hepatic arterial infusion of floxuridine, leucovorin, doxorubicin, and cisplatin for hepatocellular carcinoma: effect of hepatitis B and C viral infection on drug toxicity and patient survival. J Clin Oncol 12: 1204-1211, 1994.

4. Toyoda H, Nakano S, Kumada T, et al: The efficacy of continuous local arterial infusion of 5-fluorouracil and cisplatin through an implanted reservoir for advanced hepatocellular carcinoma. Oncology 52: 295-299, 1995.

5. Minoyama A, Yoshikawa M, Ebara M, Saisho H, Sugiura N and Ohto M: Study of repeated arterial infusion chemotherapy with a subcutaneously implanted reservoir for advanced hepatocellular carcinoma. J Gastroenterol 30: 356-366, 1995.

6. Asahara T, Itamoto T, Katayama K, et al: Adjuvant hepatic arterial infusion chemotherapy after radical hepatectomy for hepatocellular carcinoma - results of long-term follow-up. Hepatogastroenterology 46: 1042-1048, 1999.

7. Okuda K, Tanaka M, Shibata J, et al: Hepatic arterial infusion chemotherapy with continuous low dose administration of cisplatin and 5-fluorouracil for multiple recurrence of hepatocellular carcinoma after surgical treatment. Oncol Rep 6: 587-591, 1999.

8. Sumie S, Yamashita F, Ando E, Tanaka M, Yano Y, Fukumori K and Sata M: Interventional radiology for advanced hepatocellular carcinoma: Comparison of hepatic arterial infusion chemotherapy and transcatheter arterial lipiodol chemoembolization. AJR Am J Roentgenol 181: 1327-1334, 2003.

9. Ando E, Yamashita F, Tanaka M and Tanikawa K: A novel chemotherapy for advanced hepatocellular carcinoma with tumor thrombosis of the main trunk of portal vein. Cancer 79: 1890-1896, 1997.

10. Ando E, Tanaka M, Yamashita F, et al: Hepatic arterial infusion chemotherapy for advanced hepatocellular carcinoma with portal vein tumor thrombosis. Cancer 95: 588-595, 2002.

11. Child CG and Turcotte JG: Surgery and portal hypertension. In: The Liver and Portal Hypertension. Child CG (ed). WB Saunders, Philadelphia, pp49-51, 1964.

12. Ueno K, Miyazono N, Inoue H, Nishida H, Kanetsuki I and Nakajo M: Transcatheter arterial chemoembolization therapy using iodized oil for patients with unresectable hepatocellular carcinoma. Cancer 88: 1574-1581, 2000.

13. Trotti A, Colevas AD, Setser A, et al: CTCAE v3.0: development of a comprehensive grading system for the adverse effects of cancer treatment. Semin Radiat Oncol 13: 176-181, 2003.

14. Konno T: Targeting chemotherapy for hepatoma: arterial administration of anticancer drugs dissolved in Lipiodol. Eur J Cancer 28: 403-409, 1992.

15. Yoshikawa M, Saisho H, Ebara M, et al: A randomized trial of intrahepatic arterial infusion of 4'-epidoxorubicin with Lipiodol versus 4'-epidoxorubicin alone in the treatment of hepatocellular carcinoma. Cancer Chemother Phamacol 33: S149-S152, 1994.

16. Ichida $\mathrm{T}$, Kato M, Hayakawa $\mathrm{A}$, et al: Theraputic effect of a CDDP-epirubicin-Lipiodol emulsion on advanced hepatocellular carcinoma. Cancer Chemother Phamacol 33: S74-S78, 1994.

17. Akamatsu M, Ishikawa T, Shiratori Y, et al: Factors predisposing to poorly differentiated hepatocellular carcinoma and its recurrence. Hepatogastroenterology 52: 391-397, 2005.

18. Hermanek P and Sobin LH: TMN Classification of Malignant Tumors. 4th edition, Springer Verlag, Berlin, pp59-61, 1987. 